\title{
Modelo de conhecimento para mapeamento de instrumentos da gestão do conhecimento e de agentes computacionais da engenharia do conhecimento
}

Sandro Rautenberg

Professor do Depto de Ciência da
Computação/UNICENTRO.Doutor em Engenharia
e Gestão do Conhecimento - EGC/UFSC

Andrea Valéria Steil

Professora do Programa de Pós-Graduação em Engenharia e Gestão do Conhecimento EGC/UFSC e do Depto de Psicologia/UFSC

José Leomar Todesco

\author{
Professor do Programa de Pós-Graduação em \\ Engenharia e Gestão do Conhecimento - \\ EGC/UFSC
}

Este artigo, tem como base uma visão interdisciplinar da práxis das áreas de Engenharia do Conhecimento e de Gestão do Conhecimento nos processos de criação, organização, formalização, compartilhamento, aplicação e refinamento de conhecimento. Uma análise da literatura dessas áreas revelou um desconhecimento dos construtos utilizados em pesquisas e desenvolvimentos interdisciplinares e a necessidade de um modelo para a convergência de construtos inerentes. Discute-se um modelo de conhecimento, para mapear a utilização de Agentes Computacionais da Engenharia do Conhecimento em Instrumentos da Gestão do Conhecimento. Via Engenharia de Ontologias, o modelo proposto teve sua viabilidade técnica comprovada em dois estudos de caso, confirmando sua capacidade para organizar, formalizar e compartilhar conhecimento já estabelecido; e criar conhecimento novo, não estabelecido academicamente, para ser aplicado e refinado. O modelo foi verificado por especialistas, que apontaram, dentre os resultados: i) um conjunto de questões que norteiam o entendimento interdisciplinar de objetos de investigação da Engenharia e da Gestão do Conhecimento; e ii) uma forma de integração de unidades de conhecimento inerente ao 
projeto de Agentes Computacionais da Engenharia do Conhecimento como soluções tecnológicas mais aderentes aos Instrumentos da Gestão do Conhecimento.

Palavras-chave: Modelo de conhecimento; Agentes computacionais da engenharia do conhecimento; Instrumentos da gestão do conhecimento; Engenharia e gestão do conhecimento; Inteligência artificial; Ontologia para engenharia e Gestão do conhecimento.

\section{Knowledge model for mapping knowledge management instruments and knowledge engineering computational agents}

This article is based on an interdisciplinary view of the Knowledge Engineering and Knowledge Management practices in the knowledge processes (creation, organization, formalization, sharing, application and refinement). A literature review of these areas revealed some misunderstanding of the constructs used in interdisciplinary researches and developments, and the necessity of a model for the convergence of involved constructs. So, it is discussed a knowledge model for mapping the use of Knowledge Engineering Computational Agents in Knowledge Management Instruments. By the Ontological Engineering, the technical feasibility of the developed model has been proved in two scenarios, confirming its ability to: organize, formalize and share already established knowledge, and create new knowledge, not established academically, to be applied and/or refined. The model was also verified by domain experts from the involved fields who pointed, among the results: i) a set of questions that guide the understanding of interdisciplinary research objects of the Knowledge Engineering and Knowledge Management; and ii) a form to integrate knowledge units in the design of Agents Computational Engineering Knowledge as technology solutions more adherent to Knowledge Management Instruments.

Keywords: Knowledge Model; Knowledge engineering computational agents; Knowledge management 
Modelo de conhecimento para mapeamento de instrumentos de gestão do conhecimento e de agentes computacionais da engenharia do conhecimento
Sandra Rautenberg;Andrea Valéria Steil; José Leomar Todesco

\section{instruments; Knowledge engineering and management; Artificial intelligence; Ontologies for knowledge engineering and management.}

Recebido em 23.08.2010 Aceito em 30.07.2011

\section{Introdução}

O artigo tem como base uma visão unificada da práxis das áreas da Engenharia do Conhecimento (EC) e da Gestão do Conhecimento (GC). A relação entre a EC e a GC é direta, uma vez que a EC desenvolve modelos, técnicas e ferramentas computacionais para apoiar a GC (SCHREIBER et al., 2002) em seus processos de criação, organização, formalização, compartilhamento, aplicação e refinamento do conhecimento (NISSEN, 2006). Neste cenário, a EC e a GC se configuram em áreas interdisciplinares e complementares, cuja convergência vem se acentuando nos últimos anos. Apesar dessa convergência, uma análise da literatura, de cada uma dessas áreas, revelou um desconhecimento dos conceitos utilizados de forma interdisciplinar.

Que tecnologias e técnicas podem ser empregadas na GC (METAXIOTIS et al., 2003)? O que deve ser feito para que Sistemas de Conhecimento tomem seu lugar como ferramentas avançadas na GC (LIAO, 2003)? Como combinar infraestrutura tecnológica e organizacional, no contexto de organizações baseadas no conhecimento (HOLSAPPLE, 2005)? Estes são alguns exemplos de questões já levantadas que motivam o desenvolvimento de um modelo de conhecimento da convergência de dois conceitos inerentes às áreas citadas, os Instrumentos da Gestão do Conhecimento e os Agentes Computacionais da Engenharia do Conhecimento ${ }^{1}$.

O desenvolvimento do modelo proposto encontra suporte diante alguns autores, a citar: Gottschalk (2005; 2007), Alavi e Leidner (2001), Mika e Akkermans (2005), Liao (2003), Liebowitz (2001) e Firestone (2008).

De acordo com Gottschalk (2005), associar e integrar a GC com a estratégia organizacional, aplicando Tecnologias da Informação e Comunicação (e.g. Agentes Computacionais da Engenharia do Conhecimento), a fim de alcançar vantagem competitiva, é um tópico importante e desafiador, tanto para pesquisadores, quanto para profissionais da área. A partir dessa perspectiva, a compreensão do que vem a ser a GC pelos engenheiros do conhecimento é fundamental (ALAVI; LEIDNER, 2001) para que o ferramental de sua área de atuação se torne mais aderente às necessidades da gestão organizacional (MIKA; AKKERMANS, 2005).

\footnotetext{
${ }^{1}$ A expressão Agentes Computacionais utilizada neste artigo, é baseada em Schreiber et al. (2002), restringindose seu sentido encontrado tão somente na literatura da Engenharia do Conhecimento, e nada tem haver com a expressão da forma como é usada na Inteligência Artificial Distribuída (WOOLDRIDGE; JENNINGS, 1995).
} 
No que se refere a tal assunto, Gottschalk (2007) também ressalta que a utilização eficiente de ferramentas e técnicas computacionais, para lidar com conhecimento, é algo crítico. Para o autor, muitas técnicas estão disponíveis, o que dificulta a análise e a comparação das mesmas. Além da diversidade de Agentes Computacionais da Engenharia do Conhecimento e de Instrumentos da Gestão do Conhecimento, Liao (2003) identificou um elevado grau de sobreposição da aplicação de diferentes agentes, em diferentes instrumentos. Parte desta sobreposição e da ambiguidade conceitual deriva da inexistência de um vocabulário de conceitos comuns (LIEBOWITZ, 2001; FIRESTONE, 2008). Frente ao exposto, a pergunta que norteou esta pesquisa foi: "Como estabelecer um modelo de conhecimento inerente ao interrelacionamento dos Instrumentos da Gestão do Conhecimento e dos Agentes Computacionais da Engenharia do Conhecimento?" O objetivo deste artigo é, portanto, descrever um modelo de conhecimento desenvolvido para mapear a utilização de Agentes Computacionais da Engenharia do Conhecimento, em Instrumentos da Gestão do Conhecimento.

Para expressar tal desenvolvimento, além desta seção introdutória, este artigo compreende: i) a fundamentação teórica, que apresenta o entendimento dos construtos GC, EC, Instrumentos da Gestão do Conhecimento, Agentes Computacionais da Engenharia do Conhecimento e Modelo de Conhecimento; ii) o procedimento metodológico adotado para construir o modelo de conhecimento proposto; iii) o modelo de conhecimento desenvolvido; iv) os estudos de caso da aplicação do modelo; v) os resultados alcançados a partir da opinião dos especialistas envolvidos; e vi) as considerações finais.

\section{Fundamentação teórica}

Tecnologicamente, a última década do século $X X$, foi marcada pelo uso massivo de Tecnologias de Informação e Comunicação, cujo agente principal de transformação foi a Internet, com sua forma diversificada de utilização. Este cenário colaborou para a geração de novos mecanismos de comunicação e compartilhamento de informações (ISKE; BOERSMA, 2005). Por sua vez, essa evolução contribuiu para o surgimento da disciplina de GC (HOLSAPPLE, 2005; STUDER et al., 2000).

Em termos acadêmicos, a GC é um campo multidisciplinar e jovem, com contribuições de diversos autores/escolas, gerando diferentes abordagens (MAIER, 2007). Com relação a esse aspecto, destacam-se duas abordagens muito distintas à GC, a japonesa e a americana (LLORIA, 2008). Para Argote (2005), estas abordagens são mais apropriadamente identificadas como abordagens orgânicas e computacionais da GC.

As abordagens orgânicas à GC são fortemente centradas na criação e no compartilhamento do conhecimento, na perspectiva do indivíduo para o grupo (LLORIA, 2008), por meio da utilização de meios sociais (SWAN; SCABROUGH, 2001). O conhecimento tácito é considerado central e grande parte das pesquisas investiga o papel e as características do 
contexto de interação humana, como mediadores da criação e do compartilhamento do conhecimento (VON KROGH; ICHIJO; NONAKA, 2001). Nestas abordagens, além do indivíduo, são considerados as redes sociais e os aspectos culturais da organização (ARGOTE, 2005). O conhecimento é, portanto, considerado um "processo".

Já as abordagens computacionais, são mais focadas na gestão do conhecimento por meio da maximização da utilização das Tecnologias da Informação e Comunicação (LLORIA, 2008). Estas abordagens privilegiam a codificação e o conhecimento explícito, considerando-o como um "objeto" gerenciável (SWAN; SCABROUGH, 2001; GREGOR; BENBASAT, 1999).

Considerando a base constitutiva deste trabalho, utiliza-se a abordagem computacional, cuja preocupação maior reside em transformar conteúdo informacional em conhecimento estruturado (CUNHA; CAVALCANTI, 2008). Neste contexto, entende-se que a transformação da informação em conhecimento estruturado tem alicerce no gerenciamento de unidades de conhecimento. Tais unidades são pacotes atômicos de conteúdo que, metodologicamente, são rotulados, indexados, armazenados, recuperados e manipulados (ZACK, 1999). Esses pacotes de conteúdo são utilizados por pessoas, possibilitando que o conhecimento existente em suas "cabeças" e, parcialmente, nos documentos e fontes de informação, seja organizacionalmente empregado (GOTTSCHALK, 2007; CUNHA; CAVALCANTI, 2008; AURUM; DANESHGAR; WARD, 2008). Por isso, no escopo deste artigo, a GC é compreendida como a gestão formal do conhecimento, tipicamente utilizando tecnologias avançadas (O'LEARY, 1998), para facilitar os processos de criação, organização, formalização, compartilhamento, aplicação e refinamento de conhecimento (NISSEN, 2006).

Apesar da abordagem computacional, à gestão do conhecimento, ser o foco de análise neste artigo, os autores não advocam que esta seja ou deva ser, a definição formal hegemônica de gestão do conhecimento. Pelo contrário, as diferentes perspectivas ao entendimento do conhecimento organizacional são reconhecidas e a abordagem computacional é aqui compreendida e apresentada como um apoio a uma visão mais orgânica à gestão do conhecimento.

Esta perspectiva à GC está alinhada à abordagem de Jurisica, Mylopoulos e Yu (2004), que pontuam a preocupação da GC com a utilização e a evolução do conhecimento em suas várias formas. Segundo os referidos autores, para a construção efetiva de tecnologias com o foco no auxílio à GC, precisa-se compreender como indivíduos, grupos e organizações usam o conhecimento. Como resultado desta análise, tem-se o desenvolvimento de Tecnologias da Informação e Comunicação aderentes à automação da infraestrutura de GC, auxiliando os trabalhadores do conhecimento em suas atividades (BERGERON, 2003; WIIG, 2004). Tecnologicamente, o desafio está em organizar pelo menos parte do conhecimento, visando sua disponibilização para o uso (CUNHA; CAVALCANTI, 2008; RAHE, 2009). 
Com relação a esse aspecto, Nissen (2006) argumenta que as Tecnologias da Informação e Comunicação exercem papel coadjuvante de suporte na GC, cabendo às pessoas o desempenho do papel principal de atuação no trabalho com o conhecimento. Segundo 0 autor, 0 conhecimento é nato do indivíduo e é utilizado para executar trabalho cognitivo. Contudo, ele também afirma que existem algumas tecnologias que acessam conhecimento diretamente, as oriundas da Inteligência Artificial.

De forma complementar à Nissen (2006), Shadbolt e Milton (1999) consideram importantes à GC o desenvolvimento de sistemas baseados em Inteligência Artificial. Para tais autores, muitos temas, desafios e problemas descritos pela GC são abordados em pesquisas da EC, onde engenheiros do conhecimento adaptam, testam e validam métodos e ferramentas de EC em problemas reais de GC. Em consonância a esta visão, neste trabalho, a EC é compreendida como a disciplina que fornece métodos e ferramentas para a construção de Sistemas de Conhecimento de modo sistêmico e controlável (STUDER et al., 2000).

Introduzidas as definições da GC e da EC, contextualizam-se os construtos Instrumentos da Gestão do Conhecimento e Agentes Computacionais da Engenharia do Conhecimento.

Formalmente, um Instrumento de Gestão do Conhecimento consiste em um conjunto alinhado e claramente definido de medidas organizacionais, recursos humanos e Tecnologias de Informação e Comunicação, com o propósito de intervir na base de conhecimento organizacional (MAIER, 2007). Como exemplos de Instrumentos da Gestão do Conhecimento, são enumerados: Compartilhamento de Melhores Práticas, Comunidades de Prática, Mapas de Conhecimento, Lições Aprendidas, Tutoria, E-learning, Gestão de Conteúdo ou Páginas Amarelas. Considerando a abordagem de Nissen (2006), quando um Instrumento de Gestão do Conhecimento utiliza tecnologias da Inteligência Artificial, é caracterizado o elo entre a GC e a EC, refletindo o emprego dos Agentes Computacionais da Engenharia do Conhecimento.

Em uma linha de considerações, Kendal e Creen (2007) afirmam que a Inteligência Artificial objetiva dotar os computadores com habilidades humanas. Geralmente, tal objetivo envolve a pesquisa de tecnologias novas e avançadas que, imediatamente, não são passíveis de utilização. Por outro lado, segundo tais autores, a EC visa aplicar na prática, os aspectos da Inteligência Artificial em problemas reais. Assim, entende-se que as Redes Neurais Artificiais, os Sistemas Especialistas e os Sistemas de Raciocínio Baseado em Casos são exemplos de Agentes Computacionais da Engenharia do Conhecimento, cuja definição está alinhada à visão de Schreiber et al. (2002). Segundo esses autores, agentes são indivíduos ou sistemas computacionais que, dado um domínio particular de interesse, são empregados no auxílio à execução de tarefas intensivas em conhecimento. Restringindo-se aos sistemas computacionais, tais agentes são denominados Agentes Computacionais da Engenharia do Conhecimento e são projetados em função de alguma 
tarefa de resolução de problemas, via combinação de métodos e técnicas de Inteligência Artificial e bases de conhecimento específicas (HUANG, 2009).

Interdisciplinarmente, ao se alinhar os Instrumentos da Gestão do Conhecimento e os Agentes Computacionais da Engenharia do Conhecimento, observa-se o elevado grau de sobreposição da aplicação de diferentes agentes em diferentes instrumentos (LIAO, 2003). Tal fato, reforça a necessidade de se estabelecer uma compreensão de como os Agentes Computacionais na Engenharia do Conhecimento podem ser utilizados como parte de ferramentas adequadas nos Instrumentos da Gestão do Conhecimento.

Para possibilitar a compreensão apontada, este artigo disserta sobre o desenvolvimento de um modelo de conhecimento para mapear a utilização de Agentes Computacionais da Engenharia do Conhecimento em Instrumentos da Gestão do Conhecimento baseado em ontologias. Neste trabalho, considera-se uma ontologia de acordo com a definição proposta por Borst (1997), o qual conceitua a conceitua como uma especificação formal e explícita de uma conceitualização compartilhada e alinha, esta definição, às assertivas de Devedzic (2002), Kiryakov (2006), Lacasta et al. (2006), Brazhnik (2007) e Simperl (2009). Devedzic (2002) afirma que um modelo de conhecimento deve representar os conceitos e fenômenos de um domínio particular de interesse. Por sua vez, Kiryakov (2006), Lacasta et al. (2006) e Simperl (2009) alegam que ontologias são modelos de conhecimento, pois ao mesmo tempo agrupam conceitos relevantes de um domínio e definem as relações entre conceitos, permitindo a exploração (BRAZHNIK, 2007), o compartilhamento e o reuso do conhecimento (SIMPERL, 2009). Considerando tais assertivas e particularizando um modelo de conhecimento ao contexto dos agentes e dos instrumentos, pressupõem-se como diretrizes deste trabalho:

a) O levantamento de um vocabulário que norteie o entendimento de objetos de investigação, em aplicações e em pesquisas interdisciplinares da GC e da EC;

b) O refinamento ou a explicitação de conhecimento relevante do domínio da EC a ser utilizado no projeto de Agentes Computacionais da Engenharia do Conhecimento;

c) O refinamento ou a explicitação de conhecimento relevante do domínio da GC a ser utilizado na implantação dos Instrumentos da Gestão do Conhecimento;

d) Uma forma de integração do conhecimento inerente ao projeto de Agentes Computacionais da Engenharia do Conhecimento, como soluções tecnológicas aderentes aos Instrumentos da Gestão do Conhecimento;

e) Um conjunto de insumos à prospecção de um ambiente aberto para publicação, compartilhamento e exploração do conhecimento interdisciplinar. 
Apontados os construtos e as contribuições esperadas do modelo de conhecimento proposto, a próxima seção apresenta o procedimento metodológico utilizado nesta pesquisa.

\section{Procedimentos metodológicos}

Para desenvolver o modelo proposto, a metodologia empregada foi baseada no ciclo de desenvolvimento de ontologias, uma vez que tal ciclo propicia um método sistêmico para construção e verificação de modelos de conhecimento. Ressalta-se que o procedimento adotado, segue as boas práticas das metodologias de desenvolvimento de ontologias On-toKnowledge (SURE; STUDER, 2003), METHONTOLOGY (GÓMEZ-PÉREZ; CORCHO; FERNÁNDEZ-LÓPEZ, 2004) e do guia Ontology Development 101 (NOY; MCGUINNESS, 2008), que envolvem as seguintes atividades:

a) Especificação - atividade cujo propósito é discernir sobre os custos do desenvolvimento de uma ontologia de suporte ao modelo de conhecimento;

b) Aquisição do conhecimento - atividade de maior interação com os especialistas de domínio para abstração dos elementos constitutivos do modelo;

c) Implementação - atividade de menor interação com os especialistas de domínio, reservada às tarefas de cunho técnico/tecnológico;

d) Verificação - atividade em que se resgata a interação com os especialistas de domínio, para averiguar se o modelo desenvolvido atende aos requisitos especificados.

Salienta-se que o desenvolvimento do modelo de conhecimento contou com a participação de engenheiros do conhecimento e gestores do conhecimento, como especialistas de domínio. Para definir o universo desses especialistas, utilizou-se o Portal Inovação (PORTAL..., 2009). Através dos mecanismos de busca deste portal, foi possível selecionar pesquisadores e/ou profissionais, segundo seus critérios de competência científica e/ou tecnológica, titulação e localização. Diante tais critérios, buscou-se selecionar os especialistas de domínio localizados em determinada unidade federativa, com competência científica/tecnológica em GC e/ou EC e que fossem doutores. Foram selecionados e convidados 12 (doze) especialistas que figuravam entre os mais bem ranqueados, privilegiando-se o critério de equidade numérica (seis especialistas de cada área). Cabe ressaltar que a composição deste grupo não teve o intuito de representar uma população, mas dele capturar algo significativo em relação a um objeto de estudo, o modelo de conhecimento. Dos doze especialistas selecionados, nove participaram no desenvolvimento do 
modelo e para fins de discussão dos resultados, tal conjunto é denominado "especialistas participantes".

Menciona-se, ainda, a incorporação de três especialistas que não participaram nas atividades iniciais do desenvolvimento do modelo (especificação, aquisição do conhecimento e implementação). Tal conjunto de especialistas, foi somente consultado para ratificação da opinião dos especialistas participantes, quando da atividade de verificação. Este grupo é denominado "especialistas não participantes".

\section{0 modelo de conhecimento}

Durante o desenvolvimento do modelo, o objetivo primordial perseguido foi indagar os especialistas participantes para que estes elaborassem questões pertinentes aos requisitos que o modelo de conhecimento deveria atender. Como resultado, foi possível estruturar os elementos constituintes do modelo (FIG. 1) e, principalmente, identificar as dimensões de informação que deveriam ser suportadas (FIG. 3). A forma como ocorre o mapeamento no modelo de conhecimento desenvolvido é exemplificada a seguir.

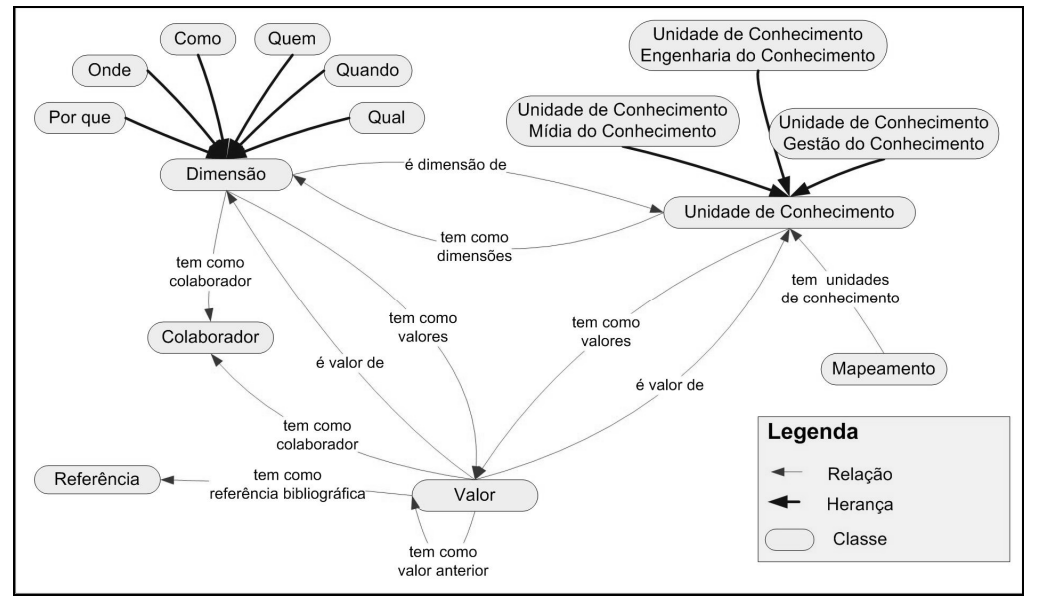

FIGURA 1 - Representação do modelo desenvolvido

Fonte: elaborado pelos autores.

Considerando os elementos "Unidade de Conhecimento", "Dimensão" e "Valor" e as relações entre os elementos "tem como valores", "é valor de", "tem como dimensões" e "é dimensão de", percebese que o mapeamento ocorre por meio das relações entre as instâncias de "Valor" para com as instâncias de "Dimensão"; as instâncias de "Valor" para com as instâncias de "Unidade de Conhecimento"; e as instâncias de "Dimensão" para com as instâncias de "Unidade de Conhecimento". 


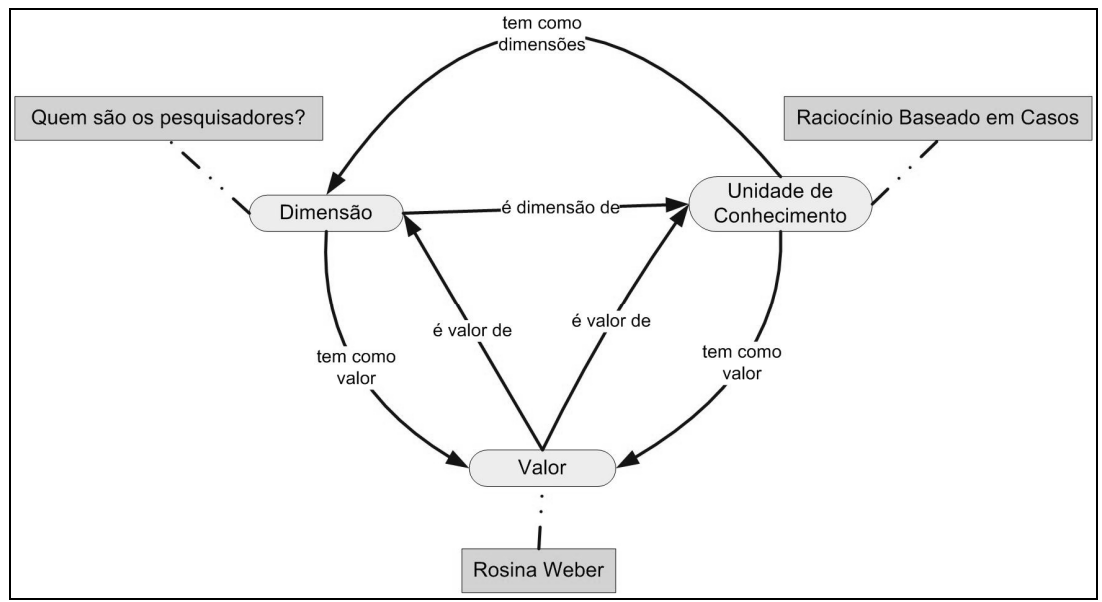

FIGURA 2 - Exemplificação do mapeamento no modelo

Fonte: elaborado pelos autores.

Exemplificando, a FIG. 2 esboça a seguinte situação: "Quem são os pesquisadores do Agente Computacional da Engenharia do Conhecimento denominado Raciocínio Baseado em Casos?". Ao simular a situação, o modelo traz como resposta a instância que representa a pesquisadora "Rosina Weber". Perante o modelo, o acesso à instância "Rosina Weber" se dá pelas seguintes circunstâncias:

1) "Quem são os pesquisadores?" representa uma instância de "Dimensão", que está relacionada à instância de "Valor", denominada "Rosina Weber" e, também, à instância de "Unidade de Conhecimento" denominada "Raciocínio Baseado em Casos";

2) Além de estar atrelada à instância de "Dimensão" "Quem são os pesquisadores?", a instância de "Valor" "Rosina Weber" também está atrelada à instância de "Unidade de Conhecimento" "Raciocínio Baseado em Casos".

Em outras palavras, "Rosina Weber" é o elo entre "Quem são os pesquisadores?" de "Raciocínio Baseado em Casos".

No exemplo, o mapeamento dos Agentes Computacionais da Engenharia do Conhecimento para com os Instrumentos da Gestão do Conhecimento ainda não é evidenciado. Para efetivar o mapeamento, considera-se que as instâncias de "Valor" devem ser compartilhadas entre duas instâncias de "Unidade de Conhecimento", uma representando um Instrumento da Gestão do Conhecimento e, outra, um Agente da Engenharia do Conhecimento. Tal fato é exemplificado na seção "5 - Estudos de Caso".

Além da estruturação do modelo de conhecimento, outro resultado que cabe ressaltar é a riqueza de dimensões de informação do modelo. Durante a atividade de "aquisição do conhecimento", foram levantadas, junto aos especialistas de domínio, 42 dimensões de informação, que permitem a realização do mapeamento entre agentes e instrumentos. Salienta-se que o 
conjunto de tais dimensões dificilmente seria abstraído de fontes de conhecimento (livros, artigos, manuais, entre outros) em sua totalidade. A Figura 3 representa as dimensões levantadas, organizadas segundo o Framework de Zachman (SOWA; ZACHMAN, 1992).

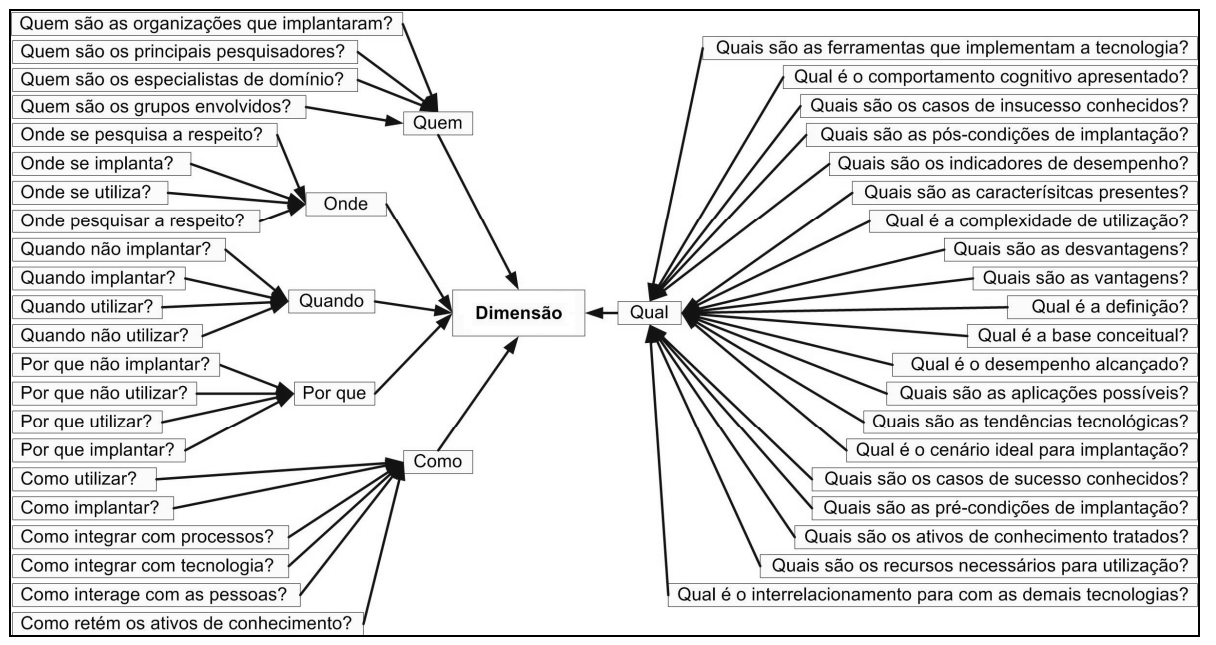

FIGURA 3 - Representação das dimensões do mapeamento

Fonte: elaborado pelos autores.

Diante à estruturação e o levantamento das dimensões de informação do modelo, foi possível configurar uma forma consistente de realizar o mapeamento entre os Instrumentos da Gestão do Conhecimento e os Agentes Computacionais da Engenharia do Conhecimento. Tal fato, foi evidenciado perante a realização de dois estudos de caso da aplicação do modelo desenvolvido. A próxima seção apresenta tais estudos de caso.

\section{Estudos de Caso}

Para verificar o modelo de conhecimento, foram propostos dois estudos de caso: a) o mapeamento do Agente Computacional da Engenharia do Conhecimento "Raciocínio Baseado em Casos (RBC)" para com o Instrumento da Gestão do Conhecimento "Lições Aprendidas (LA)"; e b) o mapeamento do Agente Computacional da Engenharia do Conhecimento "RBC" para com o Instrumento da Gestão do Conhecimento "Comunidades de Prática (CoP)".

\subsection{Mapeamento: RBC e LA}

Intencionalmente, neste estudo de caso, foram considerados alguns artigos científicos que abordam os construtos RBC e/ou LA como fontes de conhecimento. De antemão, já se sabia que estes construtos se interrelacionavam na literatura científica. A FIG. 4 evidencia 0 interrelacionamento, representando uma consulta realizada em um mecanismo de consulta de bases científicas. Na figura, são evidenciadas as consultas realizadas, onde são destacados: (A) o número de artigos 
Modelo de conhecimento para mapeamento de instrumentos de gestão do conhecimento e de agentes computacionais da engenharia do conhecimento

que citam LA como um de seus tópicos; (B) o número de artigos que citam RBC como um de seus tópicos; (C) o número de artigos que citam ambos os construtos em seus tópicos; e (D) a data da realização das consultas.

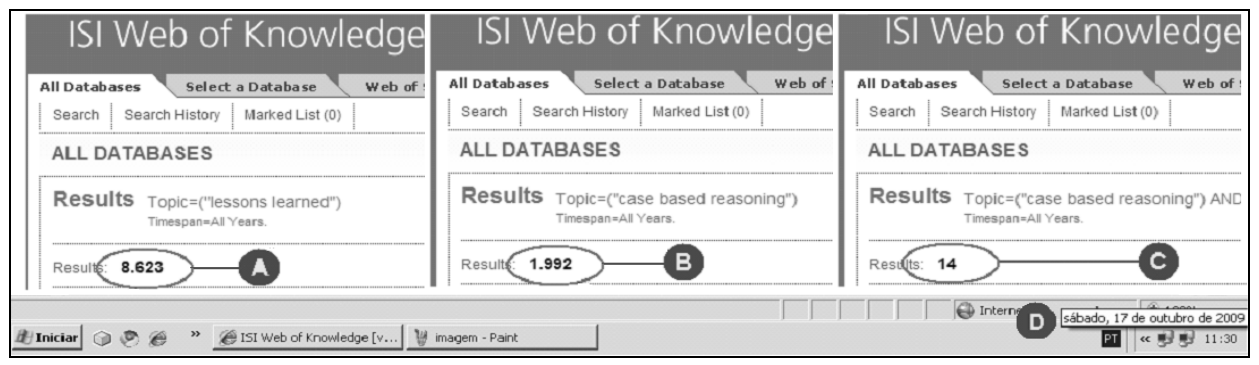

FIGURA 4 - Interrelacionamento entre RBC e LA

Fonte: adaptado de ISI (2009).

Ao simular a utilização do modelo no mapeamento do emprego de RBC em LA, das fontes de conhecimento levantadas, foram abstraídas algumas instâncias.

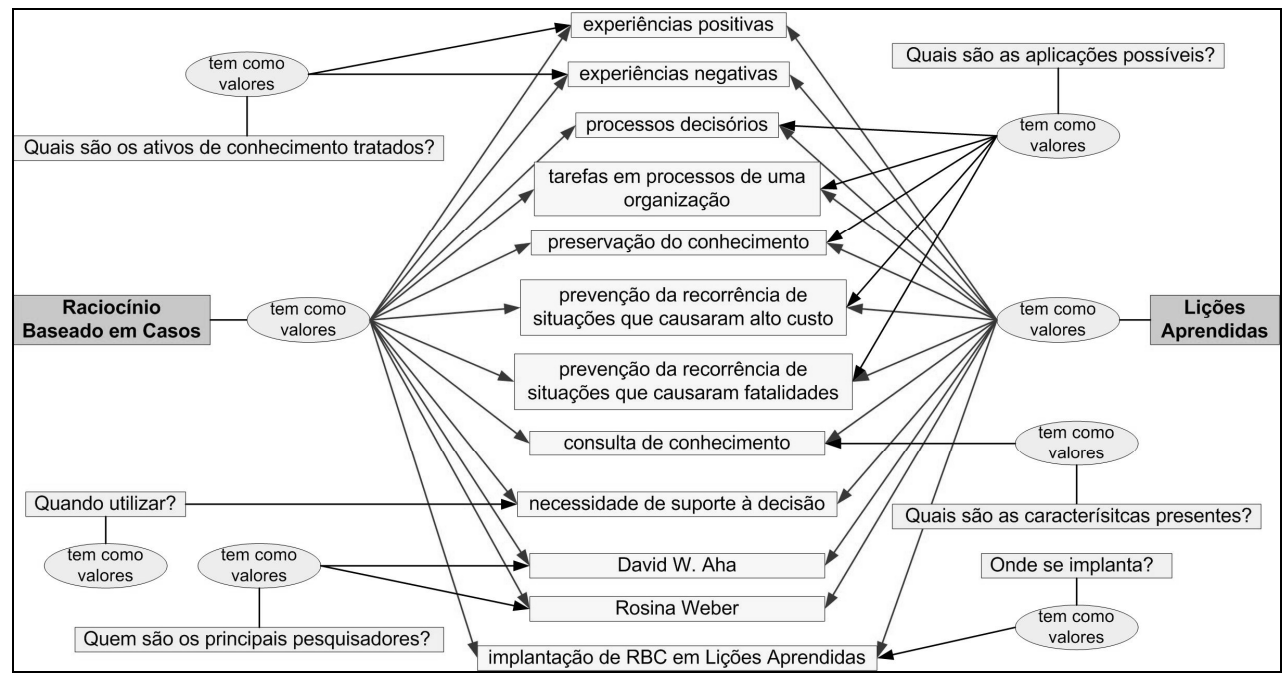

FIGURA 5 - Dimensões e valores que mapeiam RBC e LA

Fonte: elaborado pelos autores.

A FIG. 5 evidencia o mapeamento do emprego de RBC em LA, integrando a percepção de quais são as dimensões de informação, cujos valores são compartilhados entre as duas unidades de conhecimento. Ou seja, momentaneamente, de acordo com as instâncias de "Dimensão" 
"Quais são as aplicações possíveis?", RBC e LA compartilham os valores: "prevenção da recorrência de situações que causaram alto custo"; "prevenção da recorrência de situações que causaram fatalidades"; "tarefas em processos de uma organização"; "processos decisórios"; e "preservação do conhecimento". Interpretações similares, segundo a figura, podem ser feitas para as instâncias de Dimensão: "Quem são os pesquisadores?"; "Quando utilizar?"; "Onde implantar?", "Quais são as características presentes?" e "Quais são os ativos de conhecimento tratados?".

Neste estudo de caso, evidencia-se que o modelo permite incorporar indícios de mapeamento já apontados na literatura científica. Observa-se, também, um exemplo de como o modelo permite organizar, formalizar e compartilhar conhecimento explicitado cientificamente.

\subsection{Mapeamento: RBC e CoP}

Com o intuito de comprovar a reutilização de recursos no modelo (no caso, as instâncias de Valor criadas para RBC), intencionalmente, buscou-se mapear a utilização de RBC em Comunidades de Prática (CoP), como feito, anteriormente, com RBC e LA. Diante da leitura de algumas fontes de conhecimento, concluiu-se que CoP e RBC também compartilham instâncias de "Dimensão" e de "Valor", como pode ser percebido na FIG. 6.

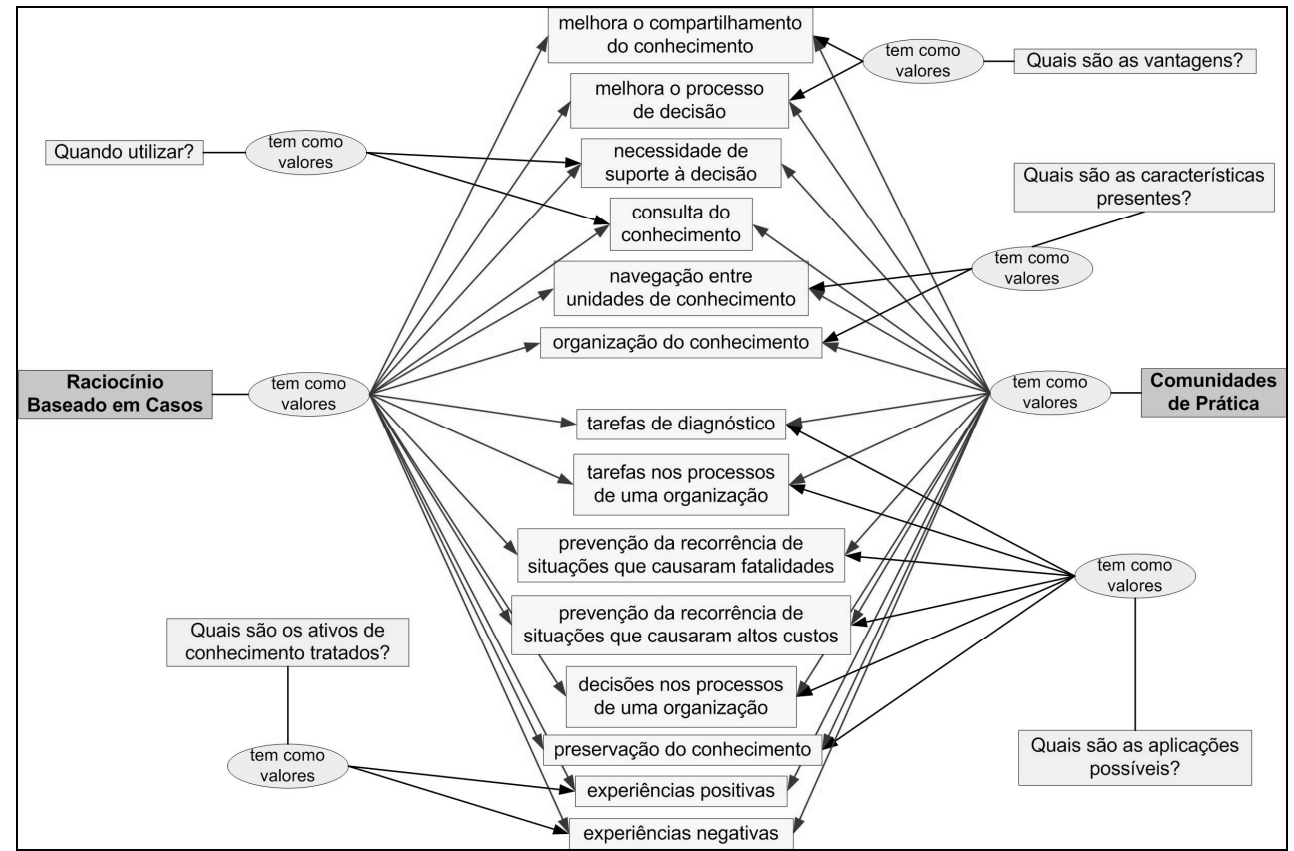

FIGURA 6 - Dimensões e valores que mapeiam RBC e CoP

Fonte: elaborado pelos autores. 


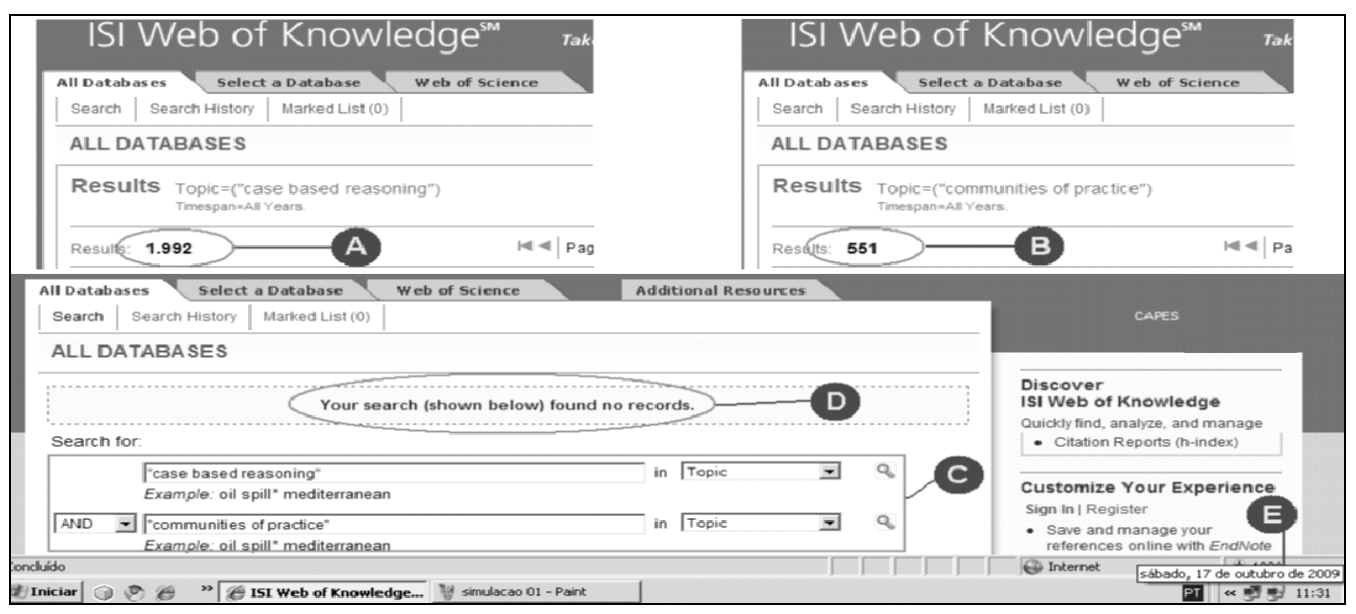

FIGURA 7 - Interrelacionamento entre RBC e CoP

Fonte: adaptado de ISI (2009).

Para comprovar o interrelacionamento neste estudo de caso, mediante 0 mecanismo de consulta em bases científicas, tentou-se comprovar o mapeamento. A FIG. 7 apresenta as consultas realizadas, onde: (A) indica o número de artigos com o tema ou palavra RBC; (B) indica o número de artigos com o tema CoP; (C) os termos consultados para a verificação do interrelacionamento; (D) a constatação que não existe um relacionamento explícito divulgado cientificamente entre os termos da consulta; (E) a data de realização das consultas.

Diante da caracterização neste cenário de aplicação, percebe-se que o modelo proposto também permite a criação de conhecimento (pelo menos, não devidamente formalizado academicamente), que pode ser aplicado e refinado.

Com os dois estudos de caso, foi possível constatar, na prática, que o modelo permite consistentemente produzir o mapeamento entre Agentes Computacionais da Engenharia do Conhecimento e Instrumentos da Gestão do Conhecimento. Porém, ainda havia a necessidade da verificação do modelo junto aos especialistas participantes e especialistas não participantes. Tal verificação é discutida a seguir na forma de resultados do modelo.

\section{Resultados}

Para verificar o modelo desenvolvido junto aos especialistas, realizou-se uma apresentação do modelo de conhecimento. Nestas apresentações, foram evidenciados: i) as definições dos Instrumentos da Gestão do Conhecimento e dos Agentes Computacionais da Engenharia do Conhecimento, ii) as diretrizes do modelo; iii) o modelo de conhecimento desenvolvido, e iv) os estudos de caso. Após as apresentações, para a captura da opinião dos especialistas participantes e dos não participantes perante o modelo desenvolvido, foi utilizado um conjunto de 13 afirmações, com uma escala de índices de 1 a 5, sendo que 1 representa 
Modelo de conhecimento para mapeamento de instrumentos de gestão do conhecimento e de agentes computacionais da engenharia do conhecimento
Sandra Rautenberg;Andrea Valéria Steil; José Leomar Todesco

"discordo completamente" e 5 representa "concordo completamente". O QUADRO 1 apresenta o conjunto das afirmações. Salienta-se que as afirmações foram formuladas, observando-se as diretrizes traçadas e inspirando-se em assertivas encontradas na literatura (MAIER, 2007; NISSEN, 2006; GASEVIC et al., 2006; GRUBER, 1993; REZGUI, 2007; BRAZHNIK, 2007; SIMPERL, 2009). Já a FIG. 8 sumariza a opinião geral dos especialistas.

\section{QUADRO 1 - Afirmações sobre o modelo desenvolvido}

1.1 AFIRMAÇÃO

1. O modelo proposto permite a organização, a formalização e a representação de conhecimento útil em projetos de Agentes Computacionais da Engenharia do Conhecimento.

2. O modelo proposto permite a organização, a formalização e a representação de conhecimento útil em projetos de Instrumentos da Gestão do Conhecimento.

3. O modelo integra conhecimento para o projeto de Agentes de Engenharia do Conhecimento, como soluções tecnológicas aderentes aos Instrumentos da Gestão do Conhecimento.

4. No modelo proposto, está circunscrito um vocabulário de termos utilizado tanto por especialistas da GC, quanto por especialistas da EC.

5. O vocabulário utilizado permite o entendimento de elementos de investigação nas pesquisas/projetos interdisciplinares da GC e da EC.

6. O modelo permite a prospecção de um ambiente aberto para publicação, compartilhamento e exploração do conhecimento interdisciplinar em comunidades de prática, por exemplo.

7. Desenvolvendo o ambiente mencionado, um requisito que deve ser atendido é o incremento do modelo proposto com novas unidades de conhecimento.

8. Desenvolvendo o ambiente mencionado, um requisito que deve ser atendido é o incremento do modelo proposto com novas dimensões de informação.

9. Desenvolvendo o ambiente mencionado, um requisito que deve ser atendido é o incremento do modelo proposto com novas informações.

10. O modelo é um guia de referência para o entendimento do relacionamento dos Instrumentos da Gestão do Conhecimento e dos Agentes Computacionais da Engenharia do Conhecimento.

11. No modelo, percebe-se o ciclo do conhecimento (NISSEN, 2006): criação, organização, formalização, compartilhamento, refinamento e aplicação.

12. O modelo é útil para os alunos, os professores, os pesquisadores e os profissionais da EC e/ou da GC.

13. O modelo atende aos anseios de interdisciplinaridade pregada no Programa de Pós-Graduação em Engenharia e Gestão do Conhecimento.

Fonte: elaborado pelos autores.

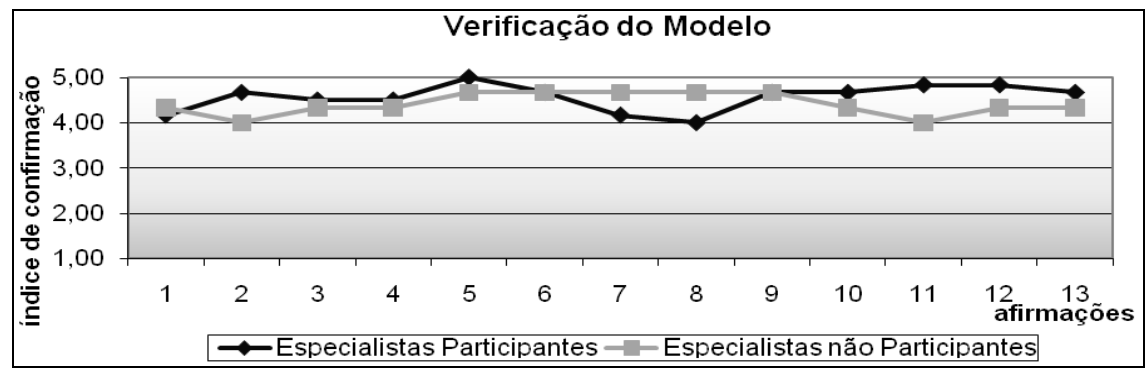

\section{FIGURA 8 - Verificação do modelo junto aos especialistas}

Fonte: elaborado pelos autores. 
Na FIG. 8, percebe-se a concordância positiva de ambos os grupos de especialistas para todas as afirmações, considerando o índice três como o ponto de neutralidade (não concordo nem discordo). Contudo, alguns especialistas registraram alguns apontamentos. Dentre os apontamentos, destacam-se:

a) Há a necessidade de uma base de conhecimento mais apurada, que permitisse maior número de simulações, o que daria mais sustentação à afirmação "3". "O modelo integra conhecimento para o projeto de Agentes de Engenharia do Conhecimento, como soluções tecnológicas aderentes aos Instrumentos da Gestão do Conhecimento";

b) Para a afirmação "No modelo proposto está circunscrito um vocabulário de termos utilizado tanto por especialistas da GC, quanto por especialistas da EC", os especialistas pontuaram que não há registros científicos de um vocabulário de termos, mas, sim, de um conjunto de questões que permitem 0 entendimento recíproco;

c) Quanto à afirmação 8 - "Desenvolvendo o ambiente mencionado, um requisito que deve ser atendido é o incremento do modelo proposto com novas dimensões de informação", os especialistas teceram críticas no sentido que o modelo parece estar completo, o que não repercute na necessidade do atendimento ao incremento de novas dimensões de informação. E, com a utilização do modelo, possivelmente, algumas dimensões de informação poderão ser pouco utilizadas, sendo um indício ao não incremento de dimensões, mas, sim, ao decremento destas;

d) Para a afirmação 11 - "No modelo se percebe o ciclo do conhecimento: criação, organização, formalização, compartilhamento, refinamento e aplicação", um especialista participante ponderou que ainda era prematuro concordar com este quesito. Haveria a necessidade de implementar o modelo computacionalmente e observar com maior propriedade sua dinâmica de utilização;

E) E, para a afirmação 13 - "O modelo atende aos anseios de interdisciplinaridade pregada no Programa de Pós-Graduação em Engenharia e Gestão do Conhecimento", apontou-se que, por o modelo se encontrar em estado embrionário, ainda é cedo para afirmar se este atende ou não ao quesito. 


\section{Considerações finais}

Este artigo apresentou o desenvolvimento de um "Modelo de conhecimento para mapeamento de Instrumentos da Gestão do Conhecimento e de Agentes Computacionais da Engenharia do Conhecimento".

De forma geral, a FIG. 9 representa o modelo desenvolvido. Nas extremidades da figura, são exemplificados os Agentes Computacionais da Engenharia do Conhecimento e os Instrumentos da Gestão do Conhecimento. Ao centro, evidenciam-se as dimensões de informação norteadoras do mapeamento proposto e que privilegiam: 1) a comunicação entre dois modelos conceituais (a convergência científica da GC e da EC); e 2) a integração de dois construtos (a convergência tecnológica dos Instrumentos da Gestão do Conhecimento e dos Agentes Computacionais da Engenharia do Conhecimento)

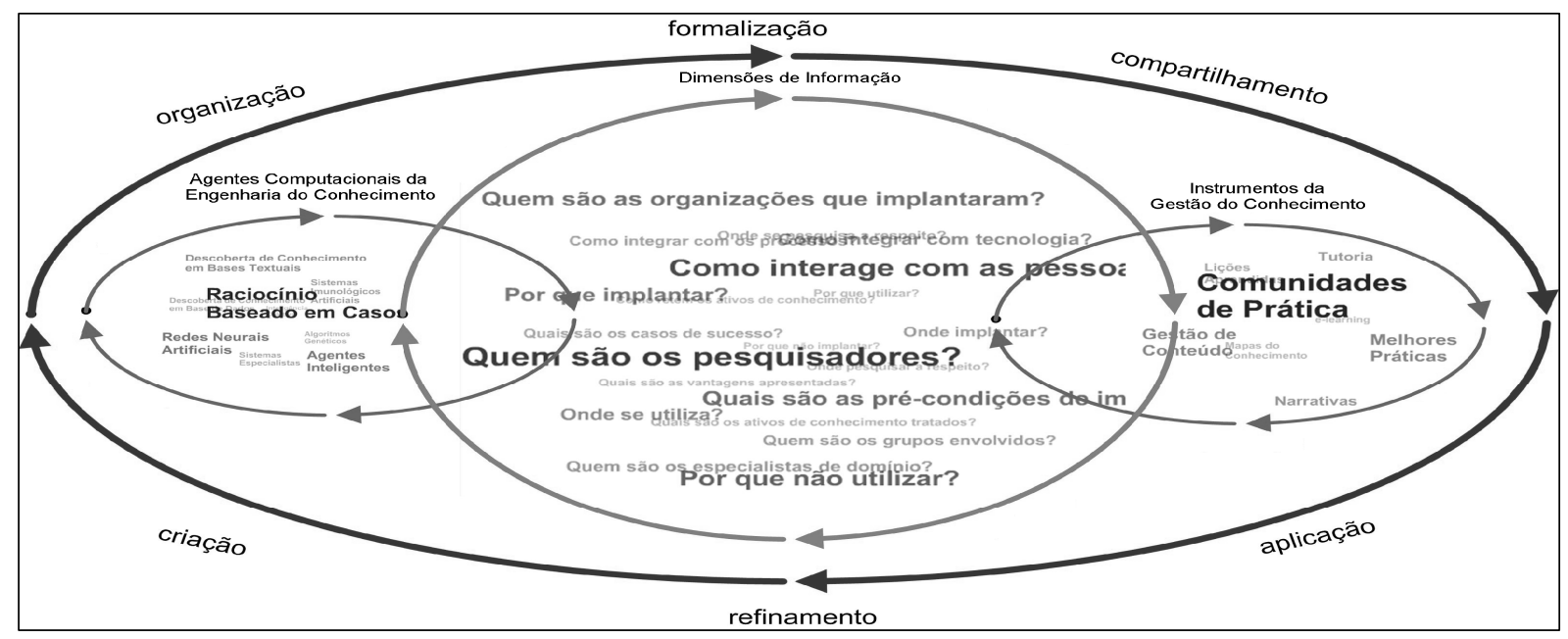

FIGURA 9 - Representação do modelo de conhecimento proposto

Fonte: elaborado pelos autores

No que tange à convergência citada, ao se explorar o conhecimento representado pelo modelo em um fluxo que perpassa os processos de criação, organização, formalização, compartilhamento, aplicação e refinamento, o modelo pode ser utilizado para auxiliar a responder questões mais abrangentes. Exemplos destas questões envolvem: i) que tecnologias e técnicas podem ser empregadas na GC?; ii) o que deve ser feito para que Sistemas de Conhecimento tomem seu lugar, como ferramentas avançadas para a GC?; ou iii) como combinar infraestrutura tecnológica e organizacional, no contexto de organizações baseadas no conhecimento?

Ao considerar tais questões, particularizando-as no contexto da EC, admite-se que o modelo proposto auxilia o engenheiro do conhecimento, sendo uma importante fonte de conhecimento à compreensão de como se 
rotula, indexa, armazena, recupera e manipula unidades de conhecimento no âmbito dos Instrumentos da Gestão do Conhecimento.

Já no lado da GC, tem-se que o modelo auxilia o gestor do conhecimento na compreensão dos paradigmas de tecnologia circunscritos em sua práxis. Ou seja, um gestor que não tem formação técnica, mas que deseja conhecer as tecnologias avançadas que podem estar abarcadas nos Instrumentos da Gestão do Conhecimento, teria uma visão geral das soluções disponibilizadas pela EC.

Por fim, ao se ponderar sobre a atuação interdisciplinar dos engenheiros e gestores do conhecimento, o modelo proposto se torna um importante meio de comunicação entre tais atores, quando do desenvolvimento de Agentes da Engenharia do Conhecimento, tecnologicamente mais aderentes aos Instrumentos da Gestão do Conhecimento. Essas constatações podem ser percebidas ao se analisar as opiniões dos especialistas na verificação do modelo, quando estes concordam positivamente.

Ressalta-se que críticas pontuais de algumas assertivas foram evidenciadas. Porém, vale destacar que durante a verificação do modelo, alguns especialistas demonstraram disposição para utilizar o modelo proposto, caso este utilizasse uma interface adequada, com possibilidade real de explorar os elementos da sua base de conhecimento. Segundo estes especialistas, a utilização de um sistema desta natureza contribuiria para materializar o modelo em uma aplicação. Neste sentido, a construção de uma interface com tal característica, é apontada como parte da evolução do presente trabalho, caracterizando-se em uma das frentes para trabalhos futuros.

Também é importante ressaltar que o modelo proposto foi testado em dois estudos de caso, que demonstraram a viabilidade de sua aplicação nos processos de criação, organização, formalização, compartilhamento, aplicação e refinamento de conhecimento interdisciplinar inerente. Contudo, para avaliar completamente o modelo, entende-se que existe a necessidade de expandir o conhecimento representado, mediante o incremento de sua base de conhecimento com novas instâncias. Como trabalho futuro, isso permitirá a realização de novos casos de estudo, possibilitando a realização e a análise de contraexemplos.

\section{Referências}

ALAVI, M.; LEIDNER, D. E. Knowledge management and knowledge management systems: conceptual foundations and research issues. MIS Quarterly, v. 25, n. 1, p. 107-136, 2001.

ARGOTE, L. Reflections on two views of managing learning and knowledge in organizations. Journal of Management Inquiry, v. 14, n. 1, p. 43-48, 2005.

AURUM, A.; DANESHGAR, F.; WARD, J. Investigating knowledge management practices in software development organisations: an Australian experience. Information and Software Technology, v. 50, n. 6, p. 511-533, 2008. 
Modelo de conhecimento para mapeamento de instrumentos de gestão do conhecimento e de agentes computacionais da engenharia do conhecimento
Sandra Rautenberg;Andrea Valéria Steil; José Leomar Todesco

BERGERON, B. P. Essentials of knowledge management. Chichester: John Wiley \& Sons, 2003.

BORST, W. N. Construction of Engineering Ontologies. Enschede, Nederland: University of Tweenty - Centre for Telematica and Information Technology, 1997.

BRAZHNIK, O. Databases and the geometry of knowledge. Data \& Knowledge Engineering, v. 61, n. 2, p. 207-227, 2007.

CUNHA, M. B.; CAVALCANTI, C. R. O. Dicionário de biblioteconomia e arquivologia. Brasília: Briquet de Lemos, 2008.

DEVEDZIC, V. Understanding Ontological Engineering. Communications of the ACM, v. 45, n. 4, p. 136-144, 2002.

FIRESTONE, J. M. On doing knowledge management. Knowledge Management Research \& Practice, v. 6, n. 1, p. 13-22, 2008.

GASEVIC, D. et al. V. Model driven architecture and ontology development. Heidelberg: Springer, 2006.

GÓMEZ-PÉREZ, A; CORCHO, O.; FENÁNDEZ-LÓPEZ, M. Ontological engineering: with examples from the areas of knowledge management, e-commerce and the semantic web. Heidelberg: Springer, 2004.

GOTTSCHALK, P. Knowledge management systems: value shop creation. London: Idea Group Inc., 2007.

GOTTSCHALK, P. Strategic knowledge management technology. London: Idea Group Inc., 2005.

GREGOR, S.; BENBASAT, I. Explanations form intelligent systems: theoretical foundations and implications for practice. MIS Quarterly, v.23, p.497-530, 1999.

GRUBER, T. Toward principles for the design of ontologies used for knowledge sharing. In: Guarino N.; Poli, R. (Eds.). International workshop on formal ontology in conceptual analysis and knowledge representation. Padova: Kluwer Academic Publishers, 1993.

HOLSAPPLE, C. W. The inseparability of modern knowledge management and computerbased technology. Journal of Knowledge Management, v. 9, n. 1, p. 42-52, 2005.

HUANG, H-C. Designing a knowledge-based system for strategic planning: a balanced scorecard prespective. Expert Systems with Applications, v. 36, n. 1, p. 209-218, 2009.

ISI Web of Knowledge. Search all Databases. Disponível em: $<$ http://apps.isiknowledge.com/UA_GeneralSearch_input.do?product=UA\&search_mode=Ge neralSearch\&SID=1F4biedMf32nfmlOCDk\&preferencesSaved=>. Acesso em: 17 out. 2009.

ISKE, P.; BOERSMA, W. Connected brains: Question and answer systems for knowledge sharing: concepts, implementation and return on investment. Journal of Knowledge Management, v. 9, n. 1, p. 126-145, 2005.

JURISICA, I.; MYLOPOULOS, J; YU, E. Ontologies for knowledge management: an information system perspective. Knowledge and Information Systems, v. 6, n. 4, p. 380-401, 2004. 
Modelo de conhecimento para mapeamento de instrumentos de gestão do conhecimento e de agentes computacionais da engenharia do conhecimento
Sandra Rautenberg;Andrea Valéria Steil; José Leomar Todesco

KENDAL, S.; CREEN, M. An introduction to knowledge engineering. Heidelberg: Springer, 2007.

KIRYAKOV, A. Ontologies for knowledge management. In: DAVIES, J. et al. (Eds.). Semantic web technologies: trends and research in ontologybased systems. Chichester: John \& Wiley, 2006. p. 115-138.

LACASTA, J. et al. A web ontology service to facilitate interoperability within a spatial data infrastructure: applicability do discovery. Data \& Knowledge Engineering, v. 63, n. 3, p. 947971, 2007.

LIAO, S. Knowledge management technologies and applications-literature review from 1995 to 2002. Expert Systems with Applications, v. 25, n. 2, p. 155-164, 2003.

LIEBOWITZ, J. Knowledge management and its link to artificial intelligence. Expert Systems with Applications, v. 20, n. 1, p. 1-6, 2001.

LLORIA, M. B. A review of the main approaches to knowledge management. Knowledge Management Research \& Practice, v. 6, n. 1, p. 77-89, 2008.

MAIER, R. Knowledge management systems information and communication technologies for knowledge management. Heidelberg: Springer, 2007.

METAXIOTIS, K. et al. J. Decision support through knowledge management: the role of the artificial intelligence. Information Management \& Computer Security, v. 11, n. 5, p. 216-221, 2003.

MIKA, P.; AKKERMANS, H. Towards a new synthesis of ontology technology and knowledge management. The Knowledge Engineering Review, v. 19, n. 4, p. 317-345, 2005.

NISSEN, M. E. Harnessing knowledge dynamics. London: Idea Group Inc., 2006.

NOY, N. F.; MCGUINNESS, D. L. Ontology development 101: a guide to creating your first ontology. Disponível em: <http://wwwksl.stanford.edu/people/dlm/papers/ontology-tutorialnoy-mcguinness.pdf $>$. Acesso em: 02 abr. 2008.

O'LEARY, D. E. Using AI in knowledge management: knowledge bases and ontologies. Intelligent Systems and Their Applications, v. 13, n. 3, p. 34-39, 1998.

PORTAL INOVAÇÃO. Home. Disponível em: <http://www.portalinovacao.mct.gov.br/pi/\#/pi>. Acesso em: 21 maio 2009.

RAHE, M, Subjectivity and cognition in knowledge management. Journal of Knowledge Management, v. 13, n. 3, p. 102-117, 2009.

REZGUI, Y. Text-based domain ontology building using Tf-Idf and metric clusters techniques. The Knowledge Engineering Review, v. 22, n. 4, p. 379-403, 2007.

SCHREIBER, G. et al. Knowledge engineering and management: the commonKADS methodology. Massachusetts: MIT Press, 2002.

SHADBOLT, N.; MILTON, N. From Knowledge Engineering to Knowledge Management. British Journal of Management, v. 10, n. 4, p. 309-322, 1999.

SIMPERL, E. Reusing ontologies on the semantic web: a feasibility study. Data \& Knowledge Engineering, v. 68, n. 10, p. 905-925, 2009. 
SOWA, J.; ZACHMAN, J. Extending and formalizing the framework for information systems architecture. IBM Systems Journal, v. 31, p. 590616, 1992.

STUDER, R. et al. Situation and perspective of knowledge engineering. In: CUENA, J et al. (Eds.). Knowledge engineering and agent technology: IOS series on frontiers in artificial intelligence and applications. Amsterdam: IOS Press, 2000.

SURE, Y.; STUDER, R. A Methodology for ontology-based knowledge management. In: DAVIES, J. et al. (Eds.). Towards the semantic web: ontology-driven knowledge management. Chichester: John Wiley \& Sons, 2003. p. 33-46.

SWAN, J; SCARBROUGH, H. Knowledge management: concepts and controversies. Journal of Management Studies, v. 38, n. 7, p. 913-921, 2001.

VON KROGH, G.; ICHIJO, K.; NONAKA, I. Facilitando a criação de conhecimento. Rio de Janeiro: Campus, 2001.

WIIG, K. M. People-focused knowledge management: how effective decision making leads to corporate success. Amsterdam: Elsevier, 2004.

WOOLDRIDGE, M; JENNINGS, N. R. Intelligent agents: theory and practice. Knowledge Engineering Review, v. 10, n. 2, p. 115-152, 1995.

ZACK, M. H. Managing codified knowledge. Sloan Management Review, v. 40, n. 4, p. 45-58, 1999. 